\title{
ARTICONF: Towards a Smart Social Media Ecosystem in a Blockchain Federated Environment*
}

\author{
Radu Prodan ${ }^{1}$, Nishant Saurabh ${ }^{1}$, Zhiming Zhao ${ }^{2}$, Kate Orton-Johnson ${ }^{3}$, \\ Antorweep Chakravorty ${ }^{4}$, Aleksandar Karadimce ${ }^{5}$, and Alexandre Ulisses ${ }^{6}$ \\ 1 Institute of Information Technology, University of Klagenfurt, Austria \\ 2 Informatics Institute, University of Amsterdam, Netherlands \\ 3 School of Social and Political Science, University of Edinburgh, UK \\ 4 Department of Electrical Engineering and Computer Science, University of \\ Stavanger, Norway \\ ${ }^{5}$ University of Information Science and Technology, Republic of North Macedonia \\ 6 MOG Technologies, Maia, Portugal
}

\begin{abstract}
The ARTICONF project funded by the European Horizon 2020 program addresses issues of trust, time-criticality and democratisation for a new generation of federated infrastructure, to fulfil the privacy, robustness, and autonomy related promises critical in proprietary social media platforms. It aims to: (1) simplify the creation of open and agile social media ecosystem with trusted participation using a two stage permissioned blockchain; (2) automatically detect interest groups and communities using graph anonymization techniques for decentralised and tokenized decision-making and reasoning; (3) elastically autoscale timecritical social media applications through an adaptive orchestrated Cloud edge-based infrastructure meeting application runtime requirements; and (4) enhance monetary inclusion in collaborative models through cognition and knowledge supply chains. We summarize the initial envisaged architecture of the ARTICONF ecosystem, the industrial pilot use cases for validating it, and the planned innovations compared to related other European research projects.
\end{abstract}

Keywords: Decentralized social media $\cdot$ privacy $\cdot$ trust $\cdot$ blockchain · semantic network · auto-scaling · Cloud and edge computing.

\section{Introduction}

Social media platforms are key technologies for next generation connectivity with the potential to shape and mobilise patterns of communication, practices of exchange and business, creation, learning and knowledge acquisition. Typically, social media platforms are centralised with a single proprietary organisation

* ARTICONF receives funding from the European Union's Horizon 2020 research and innovation program under grant agreement number 825134 . 
controlling the network. This poses critical issues of trust and governance over created and propagated content. This is particularly problematic when data breaches, at the hands of centralised intermediaries, are a regular phenomenon. In order to facilitate global reach, improved trust, and decentralised control and ownership, innovative solutions at the user level (i.e. consumers, prosumers, businesses) and the underlying social media environment level are required.

The ARTICONF project funded by the Horizon 2020 programme of the European Union researches and develops a novel set of trustworthy, resilient, and globally sustainable decentralised social media services. ARTICONF addresses issues of trust, time-criticality and democratisation for a new generation of federated infrastructure, to fulfil the privacy, robustness, and autonomy related promises that proprietary social media platforms have failed to deliver.

Objective 1: Transparent and decentralised infrastructure creation and control. The ultimate aim is to simplify transparency in the creation, integration and federation of agile decentralised social media platforms. We achieve this by creating a novel permissioned blockchain with anonymized identities with two benefits. First, it improves users control with a secure, permanent and unbreakable link to their data and controls content ownership shared further down the network through an adaptive state transition modelling. Second, it allows users content be secured from any central authority, third parties or unauthorised individuals.

Objective 2: Improved and trusted participation. ARTICONF will deliver technologies to improve trust and eliminate malicious actors in participatory exchanges throughout the collaboration lifecycle. It will research heuristics coupled with blockchain to ensure verifiable and traceable content ownership, while preserving anonymity. It will provide abstractions to characterise diverse sets of anonymised participants and to maintain their traceability and ownership activity down the network. Through these heuristics, ARTICONF will not only be able to identify bad actors, but will also improve collaboration and trust in the environment without violating users privacy. Moreover, it will simplify and optimise maintenance costs and identify users with fake profiles to prevent the spread of malicious content.

Objective 3: Democratic and tokenized decision-making. To improve democratic participatory exchanges and collaborative decision making in social media through its collective human-agent decentralised reasoning approach, ARTICONF will research heuristics to enable crowd-cooperative applications and engage with the correct audience in an anonymised ecosystem. It will also employ role-stage programming techniques and semantic abstractions to conceptualise and map the diverse geographically distributed characteristic entities. This will bring three key benefits. First, it will improve the quality of content using a collective problem solving approach with the correct subset of users. Second, it will optimise the costs of finding interest groups and communities through smart matching with precise targeting without violating users privacy and anonymity. Finally, it will provide incentivisation to the users participating in decision-making. 
Objective 4: Elastic resource provisioning. To improve efficiency for customising, provisioning and controlling distributed peer-to-peer and Cloud virtual infrastructures required by time-critical social media applications, ARTICONF aims to deliver technologies providing a self-adaptive and self-monitored infrastructure over orchestrated networked services bringing two benefits. First, it will optimise QoS performance metrics (e.g. distribution time, latency) with proximity-based geo-profiling through seamless provisioning of a customised infrastructure across multiple geographical locations. Second, it will ensure fast recovery in the presence of faults or performance drops through rapid deployment and/or migration of application resources close to problem areas.

Objective 5: Cognitive analytics for improved collaborative economy. ARTICONF develops tools to improve efficiency and inject intelligent insights into operational and mission-critical social media businesses, achieved through guided analytics with social and predictive models for consumers, prosumers and business markets. The tools will enable contextualised socially aware and spatialtemporal data aggregation, knowledge extraction, cognitive learning about users behaviour, and risk quantification for business markets. This is seamlessly coupled with distributed blockchain-based services for early alert, real-time tracking and updated data triggers for reach and engagement analysis of events. This will allow users to analyse, control and track their return-on-investment to enhance monetary inclusion in collaborative social media.

\section{Architectural design}

Given these requirements, ARTICONF researches and validates a ubiquitous social media ecosystem and platform around four important services, displayed in Fig. 1. Trust and Integration Controller (TIC), Co-located and Orchestrated Network Fabric (CONF), Semantic Model with self-adaptive and Autonomous Relevant Technology (SMART), and Tools for Analytics and Cognition (TAC).

\subsection{Trust and Integration Controller (TIC)}

TIC provides support for creating a decentralised social media interface around three modules. First, a federated consortium blockchain which provides a set of third party authentication providers for verification of each new user with a unique identity joining the network. Second, a relationship system, which is a Turing-complete programmable unit, features the transaction states between actors and allows users define conditions on data-sharing through smart contracts. Third, personal certificate authority which is a client software and manages user groups, securely shares the keys with their members, keeps records of shared keys, and encrypts shared data before broadcasting and storing it on the blockchain and the Cloud. By design, TIC provides agreement in nearly real-time between actors by applying byzantine fault tolerant consensus mechanism. 


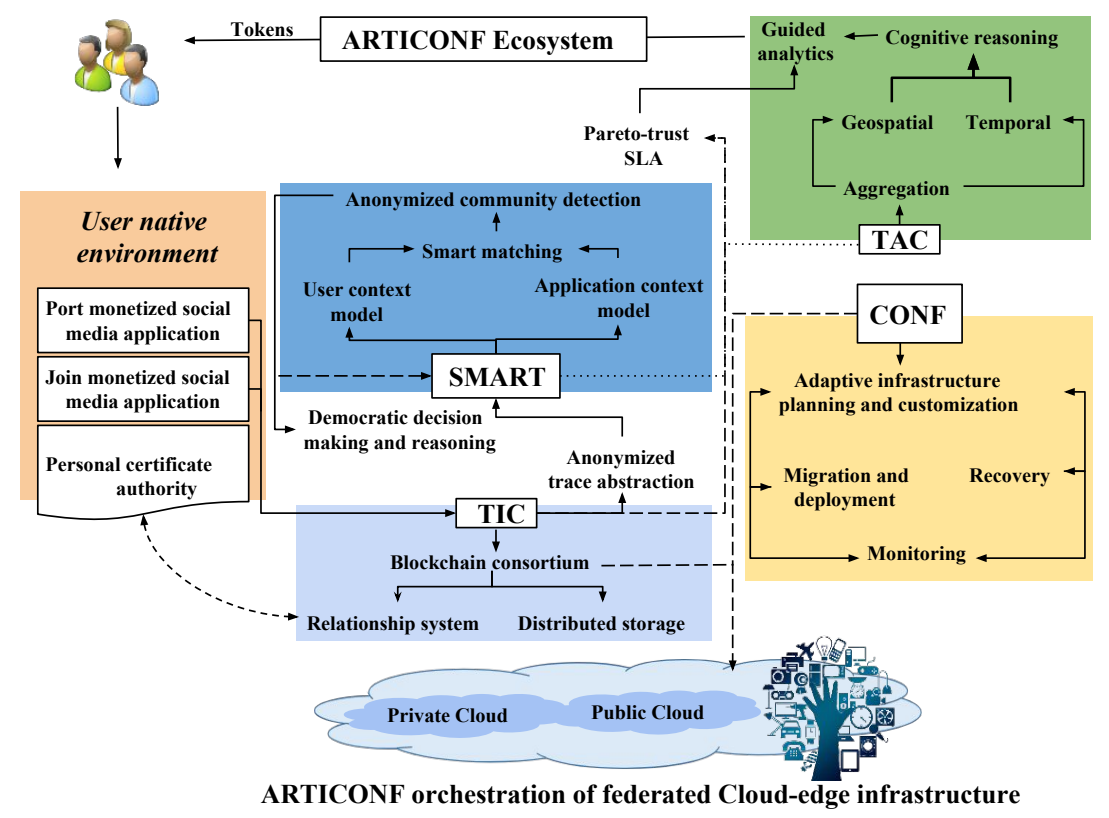

Fig. 1. ARTICONF architecture.

Permissioned consortium blockchain is the fundamental service of TIC for developing consistency, accountability and traceability of shared data. A set of third party authentication providers needs to be integrated for verification of each new user who joins the network for a given use case, providing them with a unique identity. Additionally, TIC designs and evaluates mechanisms for byzantine fault tolerant consensus algorithms able to provide agreement in nearly real-time between the different organisations, part of the permissioned blockchain network. To maintain the same federated truth for the users and improve upon the trusted collaboration, consortia of distinct organisations will equally own and maintain the blockchain seamlessly coupled with trust-based heuristics (e.g. game theoretic), and validate the transaction history for all users.

Cloud-based Big Data storage allows distributed storage of shared large data items, stored in a distributed manner with efficient indexing and traversals. This is important, as all participating nodes in the network must maintain a copy of the blockchain ensuring availability of the same version of truth. To reduce replication and network throughput, the blockchain only contains transactions logs referring to the fingerprints of the data items stored in the Cloud.

Relationship system is a Turing-complete programmable unit of the blockchain that features loops, internal states, and makes transactions with other actors. The computation executes on every participating node in the network to verify the validity of the shared transactions. This allows users define conditions on 
data sharing and use rights through smart contracts, and enable them complete control over their content. Although the blockchain removes the need for centralised authorities by entrusting the network and its peers with validation and maintenance of social interactions as transactions, it still requires mechanisms for validating user-defined conditions and successful transactions.

Certificate authority is a client software that manages user groups, securely shares the keys with their members, keeps records of shared keys, and encrypts shared data before broadcasting and storing it on the blockchain and the Cloud. It allows creation of a separate communication channel for exchange of encrypted keys between data owners and individuals with whom they share their content. It also provides identity management for users participating in the network, and ensures anonymised recording of all transactions in the blockchain, with the ability to couple them back with the original identities within local environment.

\subsection{Co-located and Orchestrated Network Fabric (CONF)}

CONF provides adaptive infrastructure provisioning for social media applications over an orchestrated network. It seamlessly integrates with the Cloud edge infrastructure, able to intelligently provision services based on abstract application service requirements, operational conditions at infrastructure level, and time-critical event triggering. The distribution of the networked infrastructure provisioned by CONF receives information from the intelligent community analytics of SMART and TAC services, and improves the infrastructure planning and customization of time-critical applications through predictive deployment, migration and failure-recovery. CONF extends the work developed in the SWITCH project [19] with support for blockchain [20] and social network business services.

Adaptive infrastructure planning driven by application-level requirements and time-critical events. CONF plans an infrastructure based on social media application requirements and adapts it in response to changing conditions, ensuring a continued and sustained satisfaction of QoS requirements.

Seamless provisioning of customised infrastructure across multiple sites. CONF provisions services and resources based on performance and reliability, taking into account locality of data sources to minimise transfers and delays, while ensuring a smooth operation of the distributed application.

Rapid deployment, migration and recovery of application services Given a distributed multi-site social media infrastructure, CONF ensures efficient deployment of application services on resources close to the active infrastructure, for fast recovery in the presence of faults or performance drops.

Self-monitoring and self-adaptation based on internal and external stimuli. The infrastructure provisioned by CONF monitors its own state, taking timely adaptation responses to failures, performance losses or other trigger conditions. CONF 
also provides interfaces required by application managers for manual adaptation, and learns how to best respond to various events based on historical data and machine learning.

\subsection{Semantic Model with Self-Adaptive Autonomous Relevant Technology (SMART)}

SMART service provide the semantic link network (SLN) abstraction model for contextualizing diverse social media actors and applications by exploiting anonymized activity traces recorded by TIC. Moreover, SMART utilizes semantic links in SLN through graph anonymisation [9] heuristics capable of detecting relevant interest group and communities without violating users privacy and anonymity. The autonomously evolving SLN model provisions SMART adaptively respond to the changing requirements, and provide inputs to CONF. SMART also researches decentralised decision making and reputation mechanisms together with TIC for solving disputes in collaborative models. To preserve the trustful environment, SMART formalizes Pareto-trust metric as a part of SLA which quantifies the quality of implicit and explicit trust-based collaboration considering various conflicting parameters, such as content accuracy, timeliness, low latency ownership verification, and high anonymity preservation.

Semantic framework for federated social media abstraction. SMART develops a conceptual model for federated social media involving large-scale entities with three abstraction levels, exploiting decentralised reasoning and relevant communities with interest groups: concrete perception of users and of associated smart objects in a global domain, structure of the perceived relationships, and communication among entities. SMART provides abstractions in representing diverse sets of participants over an anonymised social media through descriptions of classes of self-sovereign networked entities.

Autonomous and adaptive user-centric model. SMART utilises and researches role-stage programming techniques integrating various facets of social media for the design and development of a flexible, adaptive, user-centric ecosystem, involving three essential building blocks called stages, roles and agents. For this, it develops a human-agent collective-based model describing, reasoning and conceptualising consumer, prosumer and business processes at model description and at runtime. Together with TIC, SMART researches novel and efficient algorithms to preserve the autonomous interaction between diverse agents and entities with varying roles, so that one entity can rely on the others' actions.

Anonymised trace abstraction. SMART develops a framework to exploit the experiential anonymised activities embedded in blockchain. Henceforth, it provides effective and quick comparison and retrieve activity traces represented at different abstraction levels, interfaced to the TAC analytic operational support. 
Smart matching with community detection. SMART researches graph anonymization techniques for social media businesses to engage with the correct set of audience and relevant communities based on semantic abstraction and application requirements, interfaced to the CONF immersive networking support service. It also provides techniques to shift through a large number of entities, focussing on the appropriate ones through a selection of relevant, reachable and credible channels with optimised business costs.

Decentralised decision making and reputation mechanism. Through its decentralised reasoning, SMART provides opportunities for each entity irrespective of its role to be a part of decision-making process for enhancing the efficiency of collaborative business and prosumer models. Henceforth, it provisions subjectively viewed quality and immersive content creation by eradicating disputes and dissatisfaction between entities through decentralised participation. Moreover, it provides incentivisation opportunities for all social media participants for participating in such a decentralised process through its unique ripples-based reputation mechanisms, defined through evolutionary semantics abstraction and changing entity roles at varying stages during the collaborative lifecycle.

\subsection{Tools for Analytics and Cognition (TAC)}

TAC provides guided analytics by coupling with inputs from TIC to social media consumer, prosumers and businesses, aggregating contextualised data over spatial-temporal boundaries based on socio-cultural abstraction and extracting knowledge. Its goal is to provide automated cognitive learning to predict user engagement with inputs from TIC, CONF and SMART, and evaluate the risk quantification for all participants to enable, track and control the return-ofinvestment for each participant.

Geospatial, social-contextual and temporal data aggregation Aggregation and visualisation of geographical and socially diverse data are an important part of digital platforms data mining, adaptive infrastructure modelling, and management. TAC develops and integrates aggregation and data-synthesis tools using state-of-the-art technologies exploiting qualitative mapping and specification of geospatial and temporal data built upon a cross-contextualised socially aware model both internal and external to ARTICONF ecosystem.

Augmented cognition and reasoning model TAC develops robust tools for monitoring and reasoning social and cognitive states and integrate them with the ARTICONF ecosystem providing social media consumers with enhanced cognitive abilities, especially under complex collaborative participation scenarios using active and automated learning methods. Through this intelligent process, TAC reduces uncertainty, double-checks validity of information and their sources in a hostile environment, and models cross-check analytical inferences in a complex and rapidly changing social media network, requiring increased collaboration and communication. This improves collaboration amongst intelligently defined 
communities elaborating over the shared knowledge acquisition and learning, reduces biases, and gains additional benefits by drawing on the unique set of expertise and knowledge of each participant in a non-intuitive scenario.

Guided analytics for collaborative economy TAC develops an interactive interface to assist the social media consumers, prosumers and businesses inject intelligent insights in data aggregation and cognition. This guided analytics approach goes beyond a simple review of trends in sales figures by identifying techniques for increasing revenue and predicting future outcomes for an improved collaborative economy. Moreover, it provides real-time tracking of mentions, engagements, true reach and ROI. TAC is a fundamental part of ARTICONF enhancing productivity by tracking updated data triggers. TAC also allows users analyse and control their ROI through real-time cost per engagement analysis.

\section{Pilot Use Cases}

ARTICONF gathered four complementary social media use cases to pilot and validate its technology.

Crowd journalism with news verification is an application providing opportunities to independent journalists and news broadcasting industry to create content outside mainstream media by gathering crowdsourced news with public participation. Two of the main challenges faced by application providers are to validate the crowdsourced news with a requirement to find precise and trustworthy participation avoiding fake news, and to provision time-critical infrastructure resources closer to news location.

Car sharing is a form of person-to-person lending or collaborative consumption, part of the sharing economy, whereby car owners rent their vehicles to other people for short periods of time. Two challenges faced by this application are low public awareness on shared mobility, and geographical constraints with precise route, types offering precise planning, reliability and optimised business costs.

Co-creation of financial value with video is a collaborative platform for publishing and subscribing to online videos, allowing non-professional users to record videos, share them on platforms and earn rewards when their video gets watched. Two challenges faced by this application are the contextualised and thematic search of audio-visual metadata in a large video library, and the security and privacy of a scalable business model that rewards users for their interactions, including but not limited to content generation.

Smart energy uses a peer-to-peer monetised utility platform to reduce the energy bill of the prosumers by stimulating energy sharing and demand response, such that with the increasing installation of distributed generation at the demand side, more consumers become prosumers and can both generate and consume energy. 
Such human-agent models face two challenges: lack of intelligent techniques to identify the behavioural convergence of the prosumer decisions over a specific smart appliance, and lack of efficient data management plan to keep track of the amount of energy produced by each user for efficient rewards allocation.

\section{Social media application development and integration}

The TIC, CONF, SMART and TAC services are integrated part of a single coherent ARTICONF social media development platform, depicted in detail in Fig. 1. Each service encapsulates part of the overall functionality of the platform, ensuring a high-level of modularity. We aim to define a high-level abstract and generic application-programming interface between each service to ensure portability and sustainability, so that new implementations works with the rest as the technology evolves. The application development occurs in three phases, each phase consisting of seven steps.

Collaborative application porting has seven steps:

1. Social media application providers begin by porting their application using TIC, configuring their suitability and integration to the underlying permissioned blockchain;

2. Providers define application and event scenarios, scale, goals and trust requirements for performance and crowd-cooperative participation.

3. SMART service exploits specific requirements to contextualize application's trust and time-critical constraints and map them onto self-organized semantic link network (SLN) data model allowing the ecosystem to link applications with similar characteristics;

4. SMART interacts with turing-complete programmable unit of TIC to obtain the logged transactional activities of anonymised users and map them onto SLN model of the ecosystem;

5. SMART triggers the CONF's network orchestrator with the virtual infrastructure hosting the application by providing inputs with regards to precise targeting of users and communities with customised geo-profiling;

6. SMART configures TAC by providing inputs to aggregation, cognition and learning modules that analyse the behaviour and engagement of the application and social media actors, diagnose performance risks, and provide guided analytics to consumer prosumers and application providers to improve collaboration and return of investment (ROI);

7. SMART provide a decision making and reasoning model integrated to TIC's consensus mechanism based on the automatically evolving SLN based contextualization model, provided constraints, exploiting diverse cognitive states of all social objects and their characteristics generated within the SMART environment, throughout the ARTICONF ecosystem lifecycle. 


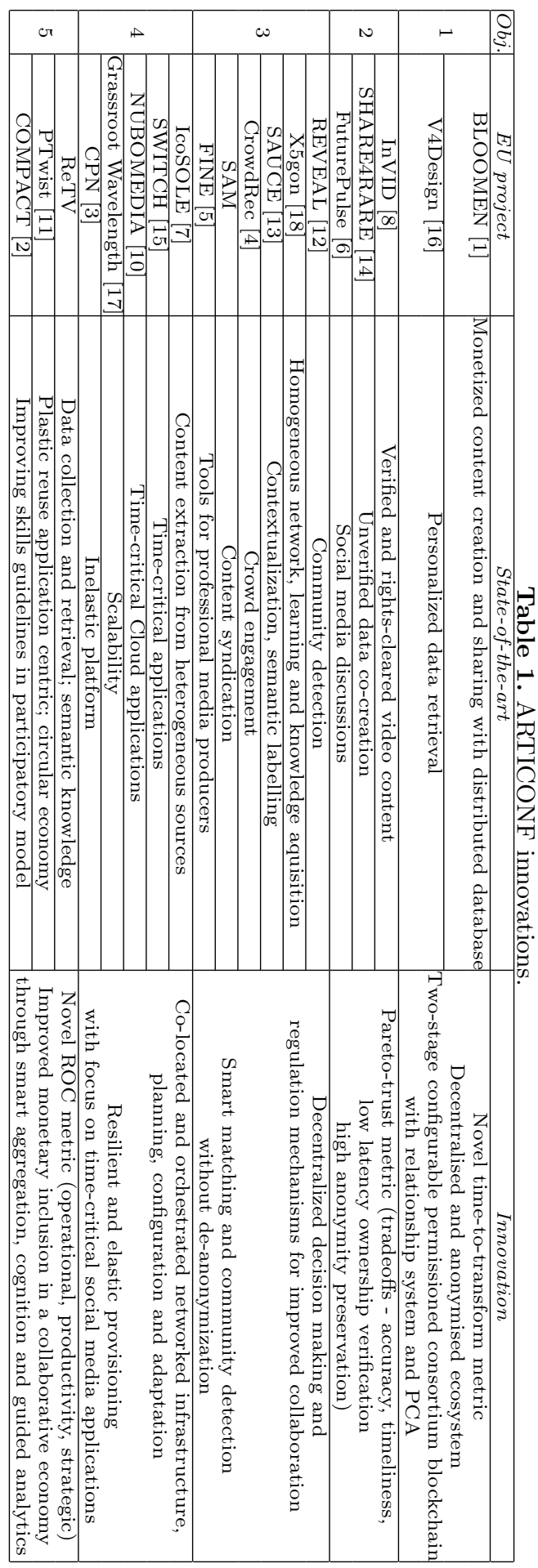


Collaborative elastic provisioning has three steps:

1. CONF receives application specification inputs from the SMART distributed knowledge base and customises a microservices-based virtual infrastructure based on the applications requirements;

2. CONF interacts with SMART and TIC to obtain information about the available and trusted Cloud edge resources obtained from subscribed infrastructure providers or users in the permissioned consortium, and creates a customised infrastructure that best meets the application needs;

3. CONF provisions the planned virtual infrastructure on selected edge providers closest to the event location, deploys all applications in cooperation with SMART, and finally schedules the execution of the application.

Application execution has two steps:

1. Through its byzantine consensus approach to maintain truth, TIC monitors the trusted behaviour of actors transaction over execution of the runtime system. It provides dynamic information to SMART and TAC, and activates the analytics and learning components in TAC for cognition and knowledge extraction via its deployed services and diagnose the runtime status when events related to cooperation, performance and security occur;

2. TIC in cooperation with SMART decides when the system is in danger of trust violations, drops in performance, or failures of the application or host infrastructure. It then invokes the infrastructure control interface provided by CONF to adapt the infrastructure accordingly. TIC records the control and adaptation solutions of CONF and SMART, further used by TAC for analysis and guided analytics.

\section{Innovation}

ARTICONF innovative solutions to problems not yet covered in sufficient depth by EU-funded projects, as summarised in Table 1.

\section{Conclusions}

We presented the ARTICONF project and its envisioned architecture, researching and developing a novel social media ecosystem and development platform focusing on achieving a major breakthrough in transparent, decentralised infrastructure creation for social media networks; with anonymous trusted participation and democratic decision-making with underlying permissioned blockchain, peer-to-peer, and elastic Cloud edge-based technologies. The project plans to validate its results on a carefully selected set of industrial applications targeting crowd journalism with news verifications, car sharing, co-creation of financial value with videos, and smart energy sharing. The project started in January 2019 and expects to achieve its first prototype results by 2020 . 


\section{References}

1. BLOOMEN: Blockchain for creative work. http://bloomen.io/

2. COMPACT: Bridging western and eastern europe for better social media and mainstream media convergence. http://compact-media.eu/

3. CPN: Content personalisation network. https://www.projectcpn.eu/

4. CrowdRec: Crowd-powered recommendation for continuous digital media. http://www.crowdrec.eu/

5. FINE: Freeview immersive networked experience. http://www.projectfine.eu/

6. FuturePulse: http://www.futurepulse.eu/

7. ICoSOLE: Immersive coverage of spatially outspread live events. http://icosole.eu/

8. InVID: In video veritas! https://www.invid-project.eu/

9. Nguyen, H.: Social Graph Anonymization. Theses, Université de Lorraine (Nov 2016), https://hal.inria.fr/tel-01403474.

10. NUBOMEDIA: The open source cloud for real-time multimedia communications. http://www.nubomedia.eu/

11. PTwist: An open platform for plastics lifecycle awareness, monetization, and sustainable innovation. http://capssi.eu/caps-projects/ptwist/

12. REVEAL: Realising education through virtual environments and augmented locations. https://revealvr.eu/

13. SAUCE: Smart asset re-use in creative environments. https://www.sauceproject.eu/

14. Share4Rare: Social media platform dedicated to rare diseases, using collective intelligence for the generation of awareness and advanced knowledge on this large group of diseases. http://capssi.eu/caps-projects/share4rare/

15. SWITCH: Software workbench for interactive, time critical and highly self-adaptive cloud applications. http://www.switchproject.eu/

16. V4Design: Visual and textual content re-purposing for architecture, design and virtual reality games. https://v4design.eu/

17. Wavelengths, G.: Highly networked grassroots community radio through a scalable digital platform. https://capssi.eu/caps-projects/grassroots-wavelengths/

18. X5GON: Cross modal, cross cultural, cross lingual, cross domain, and cross site global OER network artificial intelligence and open educational resources. https://www.x5gon.org/

19. Zhao, Z., Taal, A., Jones, A., Taylor, I., Stankovski, V., Vega, I.G., Hidalgo, F.J., Suciu, G., Ulisses, A., Ferreira, P., de Laat, C.: A software workbench for interactive, time critical and highly self-adaptive cloud applications (SWITCH). In: 2015 15th IEEE/ACM International Symposium on Cluster, Cloud and Grid Computing. IEEE (may 2015). https://doi.org/10.1109/ccgrid.2015.73

20. Zhou, H., de Laat, C., Zhao, Z.: Trustworthy cloud service level agreement enforcement with blockchain based smart contract. In: 2018 IEEE International Conference on Cloud Computing Technology and Science (CloudCom). IEEE (dec 2018). https://doi.org/10.1109/cloudcom2018.2018.00057 